\title{
Outcomes by Clinical and Molecular Features in Children With Medulloblastoma Treated With Risk-Adapted Therapy: Results of an International Phase III Trial (SJMB03)
}

\begin{abstract}
Amar Gajjar, MD¹; Giles W. Robinson, MD¹; Kyle S. Smith, PhD²; Tong Lin, PhD³; Thomas E. Merchant, DO, PhD

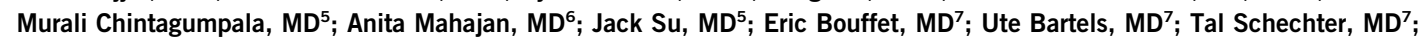
Tim Hassall, MD ${ }^{8}$; Thomas Robertson, MD $^{9}$; Wayne Nicholls, MD $^{8}$; Sridharan Gururangan, MRCP ${ }^{10}$; Kristin Schroeder, MD, MPH ${ }^{11}$; Michael Sullivan, MBChB, DCH, PhD ${ }^{12}$; Greg Wheeler, MBBS ${ }^{13}$; Jordan R. Hansford, MSc, MBBS ${ }^{12}$; Stewart J. Kellie, MBBS ${ }^{14}$; Geoffrey McCowage, MD ${ }^{14}$; Richard Cohn, MB ${ }^{15}$; Michael J. Fisher, MD ${ }^{16}$; Matthew J. Krasin, MD ${ }^{4}$; Clinton F. Stewart, PharmD ${ }^{17}$; Alberto Broniscer, MD, MS ${ }^{18}$; Ivo Buchhalter, $\mathrm{PhD}^{19}$; Ruth G. Tatevossian, MD, PhD ${ }^{20}$; Brent A. Orr, MD, PhD ${ }^{20}$; Geoff Neale, PhD ${ }^{21}$; Paul Klimo Jr, MD ${ }^{22}$; Frederick Boop, MD ${ }^{22}$; Ashok Srinivasan, MD ${ }^{23}$; Stefan M. Pfister, MD ${ }^{24}$; Richard J. Gilbertson, MD, PhD ${ }^{25}$; Arzu Onar-Thomas, $\mathrm{PhD}^{4}$; David W. Ellison, $\mathrm{MD}, \mathrm{PhD}^{2}$; and Paul A. Northcott, $\mathrm{PhD}^{2}$
\end{abstract}

PURPOSE SJMB03 (ClinicalTrials.gov identifier: NCT00085202) was a phase III risk-adapted trial that aimed to determine the frequency and clinical significance of biological variants and genetic alterations in medulloblastoma.

PATIENTS AND METHODS Patients 3-21 years old were stratified into average-risk and high-risk treatment groups based on metastatic status and extent of resection. Medulloblastomas were molecularly classified into subgroups (Wingless [WNT], Sonic Hedgehog [SHH], group 3, and group 4) and subtypes based on DNA methylation profiles and overlaid with gene mutations from next-generation sequencing. Coprimary study end points were (1) to assess the relationship between ERBB2 protein expression in tumors and progression-free survival (PFS), and (2) to estimate the frequency of mutations associated with WNT and SHH tumors. Clinical and molecular risk factors were evaluated, and the most robust were used to model new risk-classification categories.

RESULTS Three hundred thirty eligible patients with medulloblastoma were enrolled. Five-year PFS was $83.2 \%$ $(95 \% \mathrm{Cl}, 78.4$ to 88.2$)$ for average-risk patients $(\mathrm{n}=227)$ and $58.7 \%(95 \% \mathrm{Cl}, 49.8$ to 69.1$)$ for high-risk patients $(n=103)$. No association was found between ERBB2 status and PFS in the overall cohort $(P=.74)$ or when patients were stratified by clinical risk $(P=.71)$. Mutations in CTNNB1 $(96 \%), D D X 3 X(37 \%)$, and SMARCA4 (24\%) were most common in WNT tumors and PTCH1 (38\%), TP53 (21\%), and DDX3X (19\%) in SHH tumors. Methylome profiling classified 53 WNT (17.4\%), 48 SHH (15.7\%), 65 group 3 (21.3\%), and 139 group $4(45.6 \%)$ tumors. A comprehensive clinicomolecular risk factor analysis identified three low-risk groups (WNT, low-risk SHH, and low-risk combined groups 3 and 4) with excellent (5-year PFS > 90\%) and two very high-risk groups (high-risk SHH and high-risk combined groups 3 and 4) with poor survival (5-year

ASSOCIATED CONTENT

Data Supplement

Protocol

Author affiliations

and support

information (if

applicable) appear at the end of this article.

Accepted on October 29,2020 and published at ascopubs.org/journal/ jco on January 6 , 2021: DOI https://doi. org/10.1200/JCO.20. 01372 PFS $<60 \%$ ).

CONCLUSION These results establish a new risk stratification for future medulloblastoma trials.

J Clin Oncol 00. ๑ 2021 by American Society of Clinical Oncology

\section{INTRODUCTION}

Most children with medulloblastoma survive with multimodal therapy that combines maximal surgical resection, craniospinal irradiation (CSI), and chemotherapy. However, the impact of treatment-related morbidities on quality of life in survivors remains high, ${ }^{1}$ and about a quarter of patients still die of their disease. Thus, an important aspect of clinical trials must be to mitigate toxicity. However, determining which component of therapy to modify, and which patients are most likely to benefit, remains a challenge.

Traditionally, patients have been risk-stratified based on clinical characteristics to reduce the deleterious effects of therapy. Those with subtotally resected tumors (defined as residual disease of $>1.5 \mathrm{~cm}^{2}$ ) and metastatic spread of disease $\left(M_{+}\right)$are classified as high risk, whereas those with gross totally resected (GTR) or near totally resected tumors (NTR; defined as residual tumor $\leq 1.5 \mathrm{~cm}^{2}$ ) and 


\section{CONTEXT}

\section{Key Objectives}

The SJMB03 Protocol was designed to explore and define the clinical impact of biological differences found within medulloblastoma in a patient population treated on a clinically defined risk-stratified protocol.

\section{Knowledge Generated}

This study places the outcome of 330 prospectively treated patients with medulloblastoma in the context of clinical risk features, molecular subgroups, driver mutations, focal amplifications, and chromosomal aberrations and includes the latest methylation subtypes. Considering these data, we propose a novel integrated clinical and molecular risk-stratification model.

\section{Relevance}

This sophisticated risk model identifies about $40 \%$ of patients with newly diagnosed medulloblastoma who have a $>95 \%$ cure rate on SJMBO3 treatment, about $20 \%$ who have an $80 \%$ cure rate, and $40 \%$ with an unsatisfactory cure rate ranging between $30 \%$ and $60 \%$. Thus, this study lays the framework for an improved risk-stratification model and provides insight necessary for the development of less toxic and more efficacious therapy for childhood medulloblastoma.

no evidence of metastatic disease $\left(\mathrm{M}_{0}\right)$ are classified as average risk. High-risk patients receive a maximal dose of CSI with additional radiation therapy (RT) delivered to the posterior fossa, or tumor bed, and to sites of gross metastatic disease and higher or more frequent doses of chemotherapy..$^{1-3}$ By contrast, average-risk patients receive a lower CSI dose with additional RT delivered only to the posterior fossa, or tumor bed, and adjuvant chemotherapy. Using this general approach, approximately $80 \%$ of average-risk and $60 \%$ of high-risk presentations are cured..$^{1-5}$ Yet, despite similar outcomes, treatment approaches differ by the treating center or trial, and an optimized standard regimen is lacking.

Recent literature has concluded that biological differences have a major impact on outcome. Patients with histologically defined large cell/anaplastic (LC/A) medulloblastoma exhibit inferior outcomes, whereas patients with desmoplastic nodular (D/N) variants have favorable outcomes., ${ }^{6,7}$ Aberrant protein expression, such as ERBB2, or gene amplifications, such as MYC or MYCN, have been proposed as independent risk factors that negatively impact outcome. ${ }^{8-11}$ Recently, four molecular subgroups (Wingless [WNT], Sonic Hedgehog $[\mathrm{SHH}]$, group 3, and group 4) have been identified, with distinct genomic and demographic characteristics that exhibit different outcomes. ${ }^{12,13}$ Additionally, further division of these molecular subgroups into discrete subtypes has increased biologic dimensions for clinical consideration. ${ }^{14,15}$

SJMB03 (ClinicalTrials.gov identifier: NCT00085202) was a multi-institutional phase III clinical trial that emerged during a transformative era when new biologic information was rapidly accumulating. The impetus behind the trial was twofold: (1) to improve on a promising, short dose-intense therapeutic regimen from our prior study, SJMB96 (ClinicalTrials.gov identifier: NCT00003211), ${ }^{1}$ and (2) to initiate a large biology-based trial to assess the clinical utility of molecular features in a uniformly treated patient cohort.

Herein we present the results of the medulloblastoma cohort from the SJMB03 trial, including patient outcomes according to
ERBB2 protein expression status, clinical risk-based classification, and molecular features. Additionally, we identify new molecular risk features and present the first prospective outcome data based on novel medulloblastoma subtypes.

\section{PATIENTS AND METHODS}

\section{Patients and Patient Samples}

SJMB03 was a phase III clinical trial for patients 3-21 years old newly diagnosed with medulloblastoma, supratentorial primitive neuroectodermal tumor, or atypical teratoid rhabdoid tumor (Data Supplement, online only). Eligibility criteria were similar to SJMB96. ${ }^{1}$

Patients were stratified postoperatively into two risk groups (average risk and high risk). Patients with $M_{0}$ status (as indicated by magnetic resonance imaging [MRI] of the brain or spine and cytological examination of CSF) and a surgical GTR or NTR on postoperative MRI $\left(R_{0}\right)$ were assigned to average risk, whereas those with $\mathrm{M}_{+}$status $\left(\mathrm{M}_{1-3}\right.$; as indicated by MRI of the brain or spine and/or cytological examination of CSF) or residual tumor of $>1.5 \mathrm{~cm}^{2}$ on postoperative MRI $\left(R_{+}\right)$were assigned to high risk. Toxicity was monitored and graded according to Common Terminology Criteria for Adverse Events version 3.0.

Diagnoses were histologically confirmed by central pathology review. Medulloblastomas were assigned to classic, $\mathrm{D} / \mathrm{N}$, or LC/A histologic variants. Molecular analyses were done on tumor specimens and matched blood samples. Protein expression of ERBB2 was detected from freshfrozen tumor samples using immunoblotting. ${ }^{8}$ DNA was extracted from formalin-fixed paraffin-embedded (FFPE) tumor using the Maxwell rapid sample concentrator (RSC) DNA FFPE kit (\#AS1450, Promega, Madison, WI) and quantified using Qubit (Thermo Fisher Scientific, Waltham, MA) and from patient-matched peripheral WBCs using the DNeasy kit (Qiagen, Germantown, MD). RNA was extracted from snap-frozen medulloblastomas using 
STAT-60, and mRNA expression profiles were generated using the U133 Plus 2.0 microarray (Affymetrix, Santa Clara, CA) ${ }^{16}$ DNA methylation-based classification was performed using methods described previously. ${ }^{17}$ For prediction of subtypes, we implemented a random forest classifier trained on reference data sets. DNA copy number variants were inferred using the Conumee $\mathrm{R}$ package. ${ }^{18}$ Tumor and germline DNA exomes were captured using the SureSelect Human All Exon V5 (Agilent Technologies, Santa Clara, CA) platform. All next-generation sequencing (NGS) data were processed with either germline paired or unpaired analysis pipelines and curated manually to ensure consistent germline and/or somatic mutation calling. Genomic datasets included in this study can be freely explored using the online St Jude Cloud pediatric genomic data resource. ${ }^{19}$

SJMB03 was approved by the St Jude Institutional Review Board and institutional review boards at participating institutions. Patients, parents, or guardians provided written informed consent.

\section{Treatment}

Patients with average-risk disease received CSI (23.4 Gy) followed by focal RT to the primary tumor bed (total dose 55.8 Gy; 1.8 Gy daily fraction). Patients with high-risk disease received CSI (36 [M-1 $\left.]-39.6\left[\mathrm{M}_{2-3}\right] \mathrm{Gy}\right)$ followed by focal RT to the primary tumor (total dose 55.8-59.4 Gy; 1.8 Gy daily fraction). The clinical target volume margin surrounding the postoperative tumor bed was $1.0 \mathrm{~cm}$ for both risk groups. Areas of macroscopic metastatic disease $>0.5 \mathrm{~cm}$ received additional RT (total dose 50.4-59.4 Gy). Twenty-nine patients received proton-beam RT.

After a 6-week rest, patients began four cycles of highdose chemotherapy consisting of vincristine $\left(1.0 \mathrm{mg} / \mathrm{m}^{2}\right.$ once daily [max dose $2.0 \mathrm{mg}$ ] on day -4 and 6), cisplatin ( $75 \mathrm{mg} / \mathrm{m}^{2}$ once daily on day -4 ), and cyclophosphamide $\left(2 \mathrm{~g} / \mathrm{m}^{2}\right.$ once daily on days -3 and -2$)$. Each cycle was followed by stem-cell or bone-marrow rescue on day 0 . Filgrastim ( $5 \mu \mathrm{g} / \mathrm{kg}$ once daily per day) was given on day +1 until absolute neutrophil count (ANC) $>2,000$ on 2 consecutive days. The planned duration of chemotherapy was 16 weeks (4 weeks per cycle).

Treatment continued until completion, disease progression, withdrawal of consent, or unacceptable toxicity. Disease assessments were done at defined intervals until 72 months from diagnosis.

\section{Statistical Analysis}

All eligible patients who initiated RT were included. Coprimary study end points were (1) to assess the relationship between ERBB2 protein expression and progression-free survival (PFS), and (2) to estimate the frequency of mutations associated with WNT and SHH tumors. The planned sample size to detect a $15 \%$ higher PFS at 2 years for ERBB2-negative subjects compared with ERBB2-positive subjects was 123 with 5\% type I error and $80 \%$ power, taking into account the expected imbalance in the ERBB2 positivity rate between the two risk groups.

Patients with adequate tissue for methylation profiling were included in the subgroup-specific outcome analyses. Secondary biological end points aimed to describe alterations in oncogenes and tumor suppressor genes and to evaluate the relationship of these molecular features with subtype, clinical characteristics, and clinical outcome. Outcome distributions were estimated using the method of Kaplan and Meier. Logrank tests or stratified log-rank tests were used for the outcome comparisons. Cox proportional hazard models were used to estimate coefficients of risk factors in forest plots. A Fisher's exact test was used to compare distributions of categorical variables among patient groups. Statistical analyses were done using R 3.6.0.

\section{RESULTS}

\section{Study Patients}

Between September 2003 and June 2013, 413 of 416 screened patients were enrolled. Pathology review confirmed 330 (80\%) as medulloblastoma: 227 (69\%) average risk and 103 (31\%) high risk. Tolerance of therapy was very similar to what was previously described. ${ }^{1}$ Toxicities attributed to treatment that occurred in at least $5 \%$ of patients and any study-related deaths not from disease progression are listed in the Data Supplement.

Three hundred five (94\%) medulloblastomas underwent DNA methylation-based classification, assigning patients to WNT ( $n=53)$, SHH $(n=48)$, group $3(n=65)$, and group 4 ( $n=139$ ) subgroups. Two hundred ninety-three (89\%) had NGS, including 145 with matched germline DNA (Fig 1). RNA gene expression array analysis was conducted on 149 patients. Demographics and baseline characteristics are listed in Table 1.

\section{Outcomes by ERBB2 Expression Status}

The primary analysis was conducted on 120 eligible patients (84 average risk and 36 high risk). Seventy-five patients were ERBB2-positive and 45 patients ERBB2-negative. There was no difference in PFS by ERBB2 status $(71.8 \%$ [95\% Cl, 62.3 to 82.8] ERBB2-positive $v 70.6 \%$ [95\% Cl, 58.3 to 85.4 ] ERBB2-negative; $P=.74$ ) in the overall cohort (Fig 2A) or in the context of clinical risk $(P=.71$; Fig 2B).

\section{Outcomes by Clinical Risk Groups and Individual Clinical Risk Factors}

Based on a median follow-up of 8.75 years (interquartile range, 4.30 to 11.03$)$, the 5 -year PFS was $75.6 \%(95 \% \mathrm{Cl}$, 71.1 to 80.4$)$ and the 5 -year OS was $82.3 \%(95 \% \mathrm{Cl}, 78.2$ to 86.5) for the overall cohort ( $\mathrm{N}=330$; Fig 2C). The 5-year PFS was $83.2 \%(95 \% \mathrm{Cl}, 78.4$ to 88.2$)$ for average-risk and $58.7 \%(95 \% \mathrm{Cl}, 49.8$ to 69.1$)$ for high-risk patients $(P<.0001 ;$ Fig 2D). Five-year PFS based on metastatic 


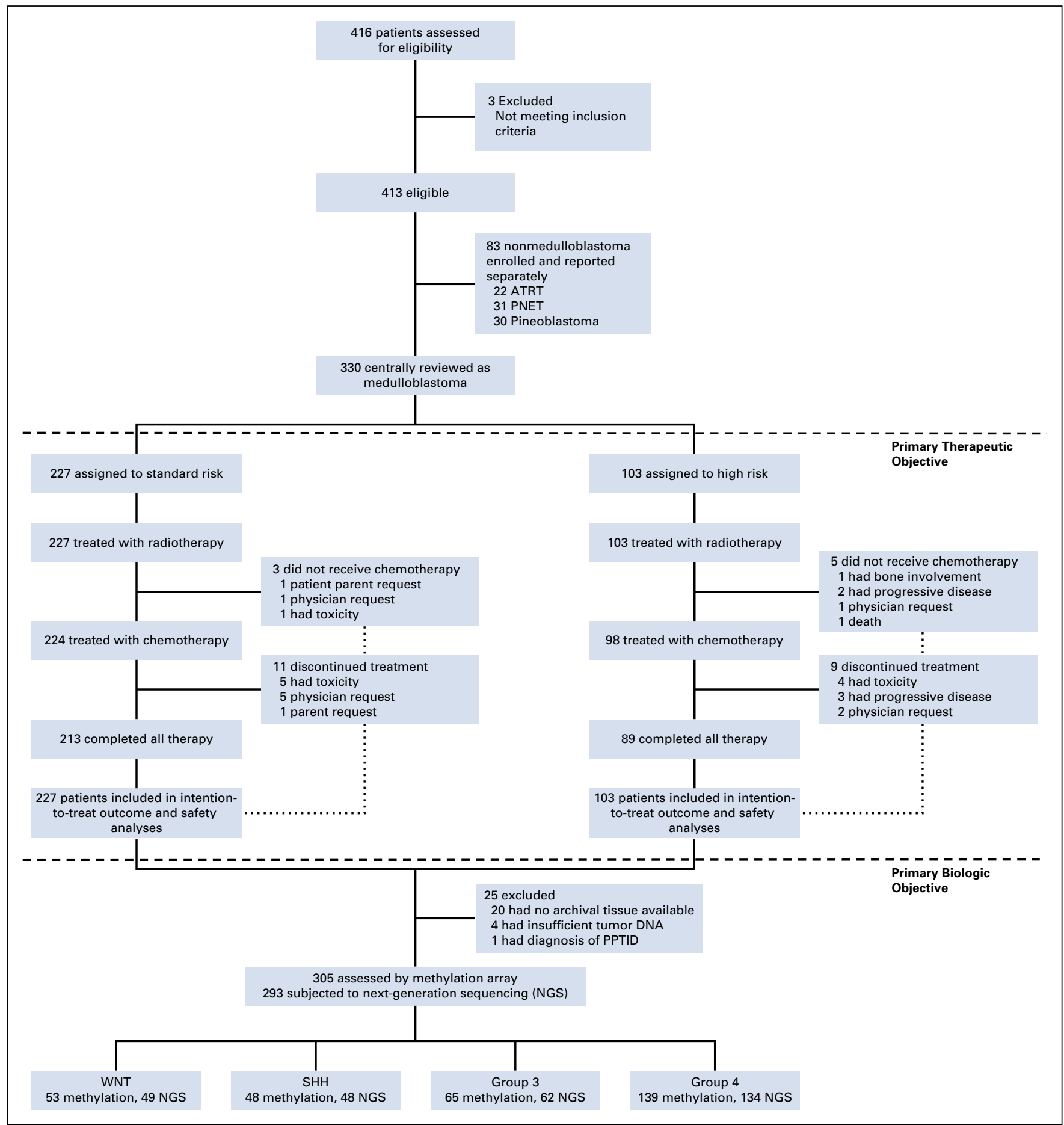

FIG 1. CONSORT diagram of the patients enrolled on the protocol and details of patients with medulloblastoma who were treated and molecularly profiled. NGS, next-generation sequencing; SHH, Sonic Hedgehog; WNT, Wingless.

status was $82.9 \%$ (95\% $\mathrm{Cl}, 78.1$ to 87.9$)$ for $\mathrm{M}_{\mathrm{O}}$ and $58.8 \%$ (95\% Cl, 49.9 to 69.4) for $\mathrm{M}_{+}$patients $(P<.0001)$ (Data Supplement).

At enrollment, 315 (96\%) patients had $\mathrm{R}_{0}$ disease and 14 (4\%) had $R_{+}$disease. $R_{0}$ subjects had better PFS compared with $R_{+}$ subjects ( $P=.0237$; Fig $2 \mathrm{E}$ ). No difference in PFS was observed between patients who underwent a GTR versus NTR
( $P=$.89; Data Supplement), and this result held when metastatic status was incorporated ( $P=.34$; Data Supplement).

\section{Outcomes by Histopathology}

Histology was significantly associated with PFS $(P<.0001$; Fig 2F). Patients with D/N histology experienced the most favorable outcomes with 5-year PFS of $92.6 \%(n=27$, 
TABLE 1. Demographic and Baseline Characteristics of the Patients

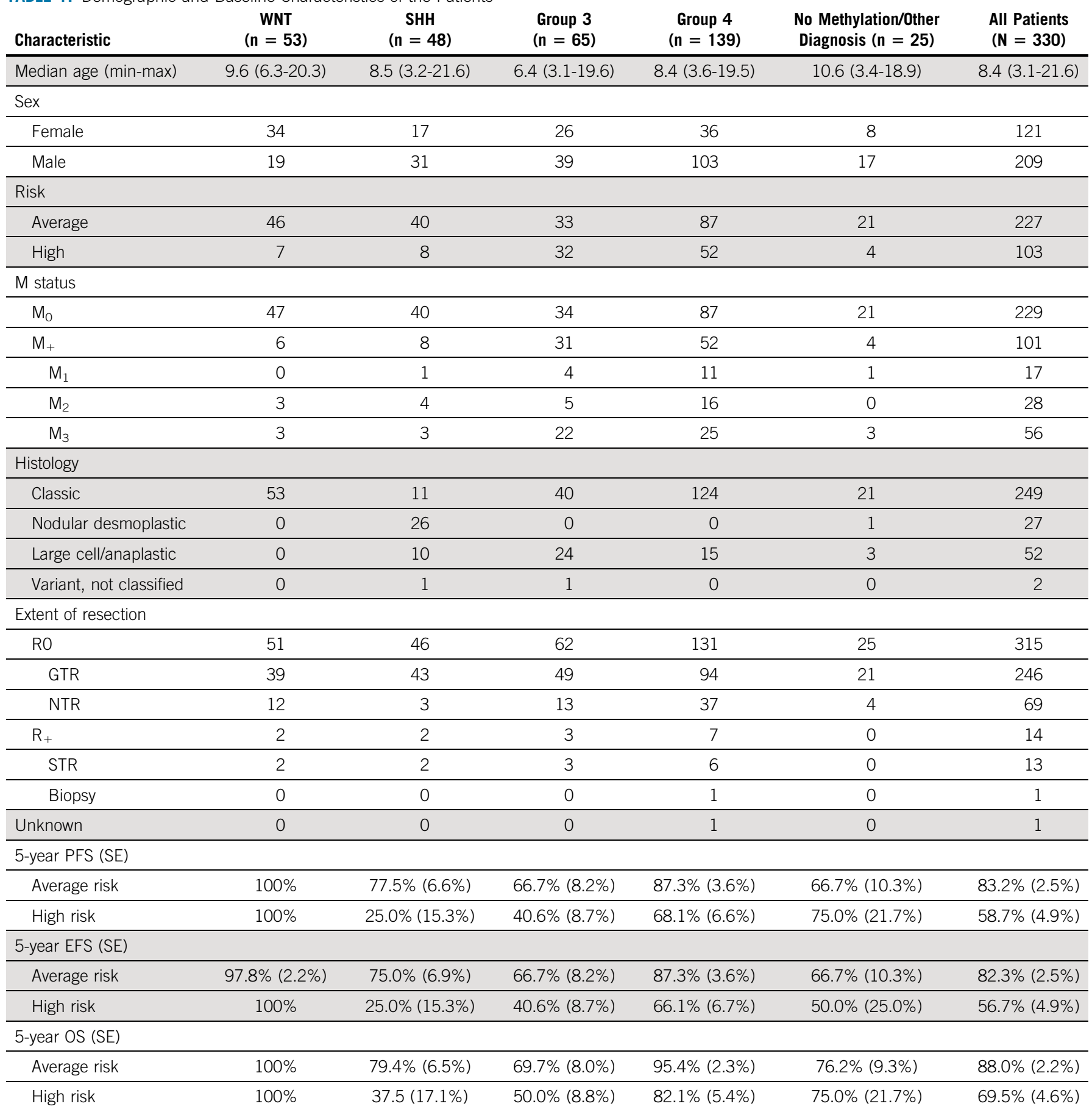

Abbreviations: EFS, event-free survival; GTR, gross totally resected; $M_{+}$, metastatic spread of disease; $M_{0}$, no evidence of metastatic disease; $M_{1-3}$, $M_{+}$ status 1, 2, or 3; NTR, near totally resected; OS, overall survival; PFS, progression-free survival; $\mathrm{R}_{0}$, average risk; $\mathrm{R}_{+}$, high risk; SHH, Sonic Hedgehog; STR, subtotally resected; WNT, Wingless.

$95 \% \mathrm{Cl}, 83.2$ to 1.00$)$, followed by those with classic histology ( $n=249$; 5-year PFS of $78.6 \%, 95 \% \mathrm{Cl}, 73.7$ to 83.9). Patients with LC/A histology had inferior outcomes ( $n=52$; PFS of $51.1 \%$ [95\% Cl, 39.1 to 66.9]). The impact of histology on PFS was maintained when patients were stratified by clinical risk ( $P=.0003$; Data Supplement).

\section{Outcomes by Molecular Subgroup}

Subgroup representation varied by risk group (Fig 3A). DNA methylation subgroups, putative driver gene alterations, and chromosomal gains and losses are summarized by molecular subgroup in Figures 3B and $3 \mathrm{C}$ 


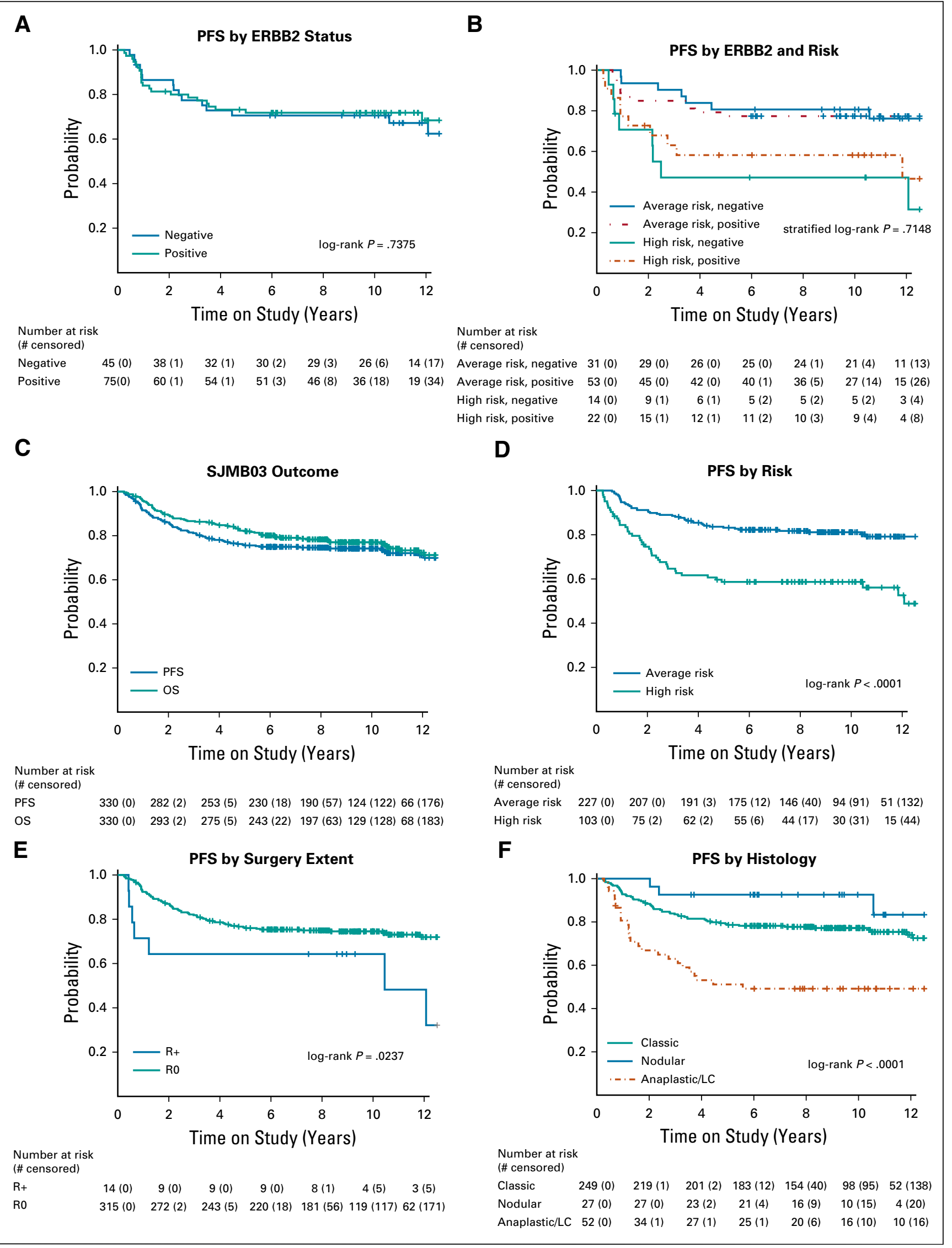

FIG 2. Survival analysis by ERBB2 and clinical risk: PFS (A) by ERBB2 status, (B) ERBB2 and risk, (C) PFS and OS of all patients with medulloblastoma, (D) PFS by risk, (E) by extent of resection, and (F) by histology. OS, overall survival; PFS, progression-free survival.

6 ㄷ 2021 by American Society of Clinical Oncology 
Among average-risk patients, 5-year PFS rates were 100\% for the WNT subgroup, $77.5 \%(95 \% \mathrm{Cl}, 65.6$ to 91.6$)$ for $\mathrm{SHH}$, $66.7 \%(95 \% \mathrm{Cl}, 52.4$ to 84.9$)$ for group 3, and $87.3 \%(95 \% \mathrm{Cl}$, 80.5 to 94.6$)$ for group 4 ( $P=.001$; Fig 4A). Among high-risk patients, 5-year PFS rates were 100\% for the WNT subgroup, $25 \%$ (95\% Cl, 7.5 to 83.0) for $\mathrm{SHH}, 40.6 \%$ (95\% Cl, 26.7 to 61.8) for group 3, and $68.1 \%(95 \% \mathrm{Cl}, 56.3$ to 82.3$)$ for group 4 $(P=.0017$; Fig 4B). Stratification by clinical risk within subgroups demonstrated inferior outcomes for high-risk patients in each subgroup except WNT (Figs 4C-F).

\section{WNT Subgroup}

The majority ( 45 of $53,85 \%$ ) of WNT tumors were midline and within the fourth ventricle. The remainder (8 of 53, 15\%) extended from the fourth ventricle to the cerebellar pontine angle. Seven (13\%) were high risk, six with metastatic disease.

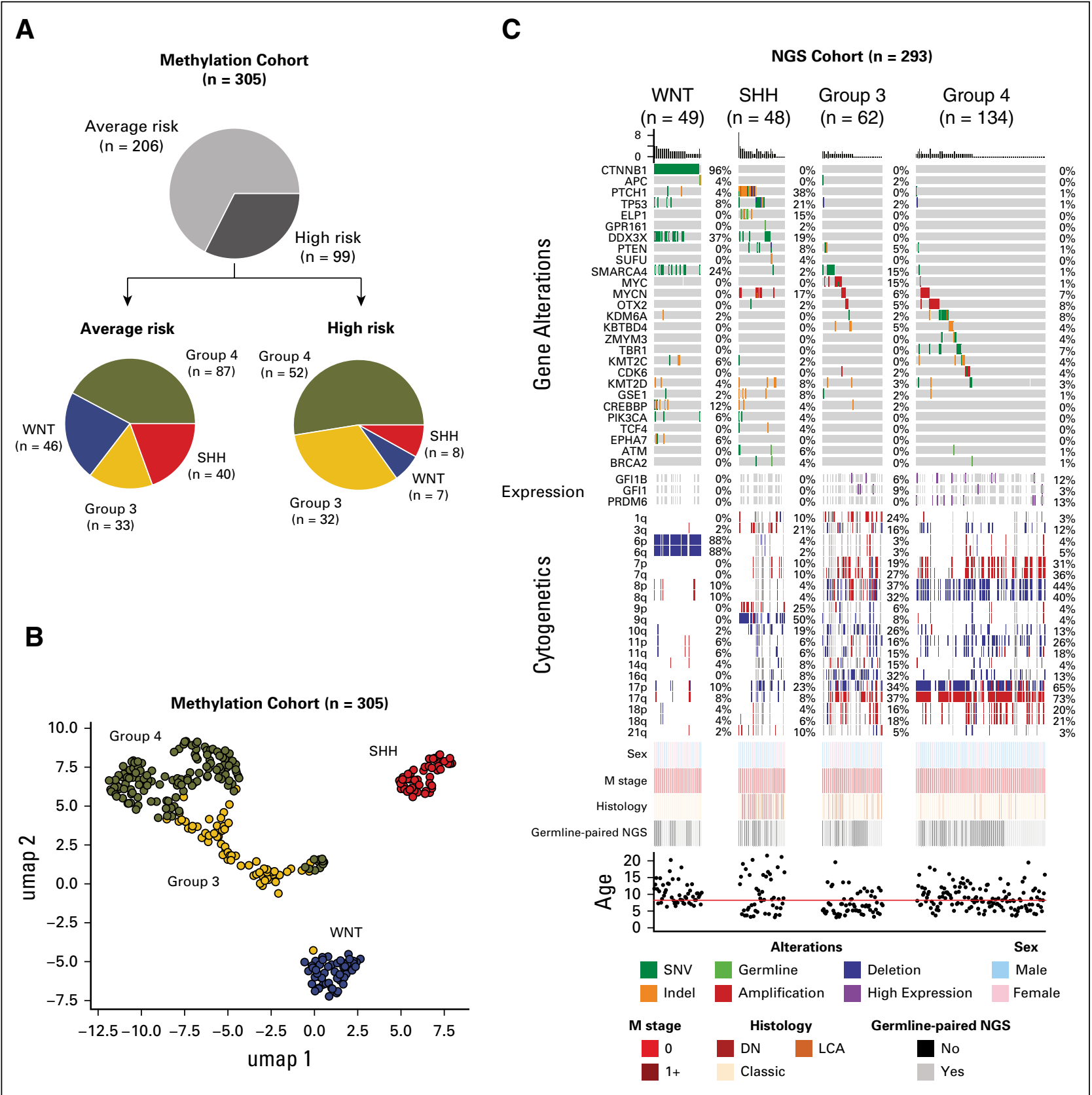

FIG 3. Genomic landscape of pediatric patients with medulloblastoma: (A) Clinical risk (AR and HR) and distribution of molecular subgroups within each risk category, (B) UMAP scatter plot based on weighted Pearson correlation of DNA methylation beta values showing the distribution of consensus medulloblastoma subgroups $(n=305)$, and $(C)$ oncoprint summarizing recurrently altered genes, frequent cytogenetic events, and sample annotation according to medulloblastoma subgroup $(n=293)$. AR, average risk; DN, desmoplastic nodular; HR, high risk; LCA, large cell anaplastic; NGS, next-generation sequencing; SHH, Sonic Hedgehog; UMAP, uniform manifold approximation and projection; WNT, Wingless. 


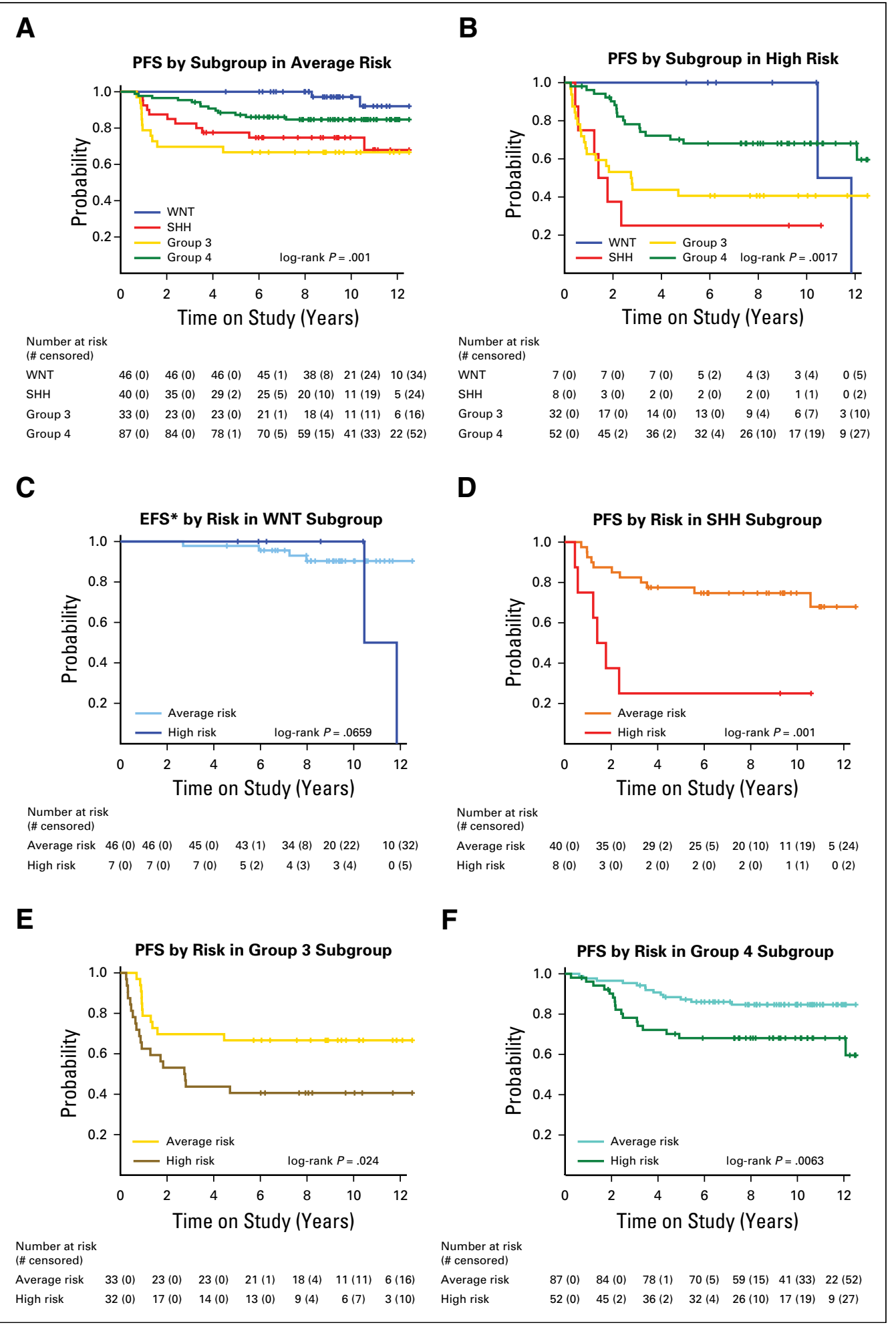

FIG 4. Survival analysis by molecular subgroup and subtype: PFS (A) by subgroup in average risk, (B) by subgroup in high risk, (C) EFS by risk in the WNT subgroup, (D) by risk in the SHH subgroup, (E) by risk in the group 3 subgroup, (F) by risk in the group 4 subgroup, $(\mathrm{G})$ by SHH subtype, and $(\mathrm{H})$ by group 3 or group 4 subtype. EFS, event-free survival; PFS, progression-free survival; SHH, Sonic Hedgehog; WNT, Wingless. 


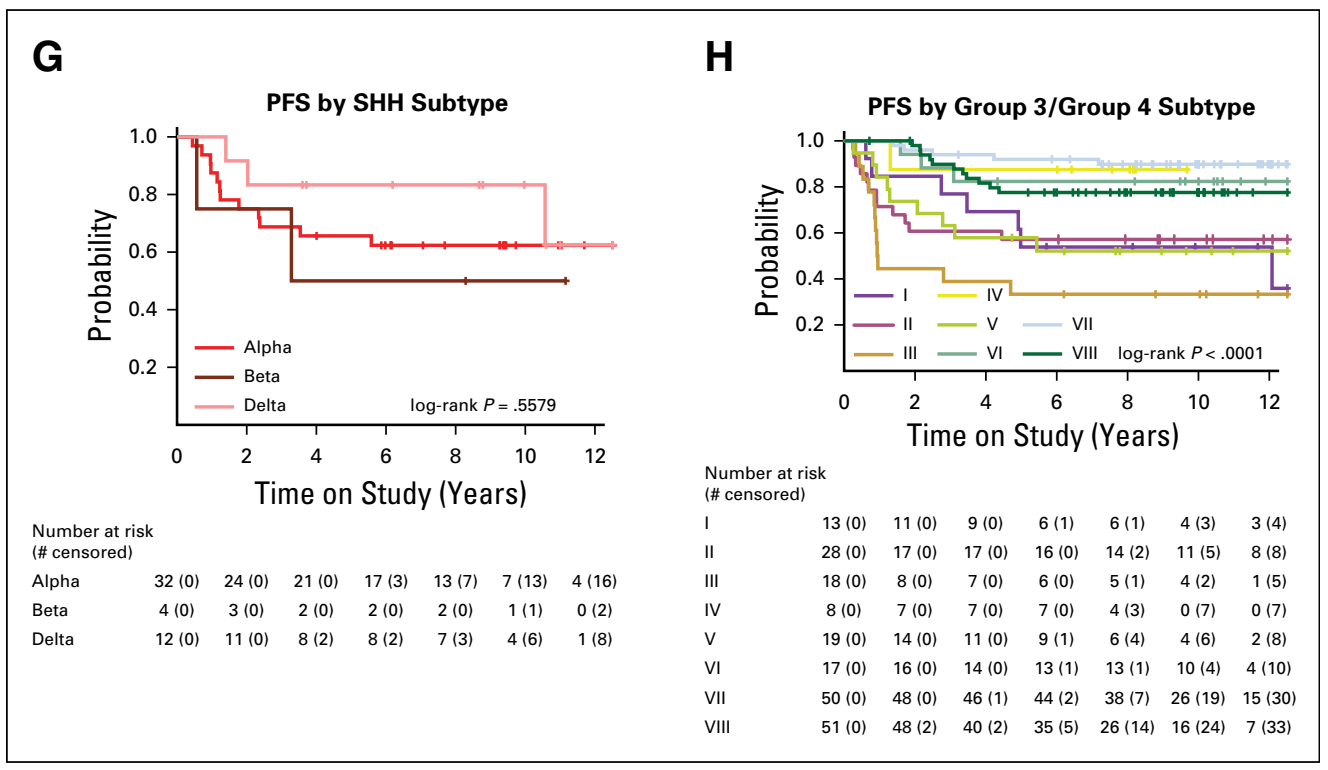

FIG 4. (Continued).

The most frequent mutations were CTNNB1 (96\%), DDX3X (37\%), SMARCA4 (24\%), and CREBBP (12\%) (Fig 3C). Monosomy chromosome 6 was detected in $89 \%$ of WNT tumors. Mutational frequencies were similar between the initial Pediatric Cancer Genome Project (PCGP) WNT cohort $(n=10)$ and the expanded (non-PCGP) WNT cohort $(n=$ 39), except for TP53 mutations that were not detected in the initial cohort (Table 2, all $P>$.10).

Although 5-year PFS and OS was 100\%, four late deaths occurred in patients with WNT. One patient developed pulmonary fibrosis and died 8.3 years from diagnosis. Additionally, four patients with WNT developed second malignancies at a median time of 7.0 years from diagnosis (range, 2.7-10.5 years) and three died. One had a confirmed germline APC mutation (Data Supplement).

TABLE 2. Common Mutations in WNT and SHH Subgroups

\begin{tabular}{lcccc} 
Gene & $\begin{array}{c}\text { PCGP WNT } \\
(\mathbf{n}=\mathbf{1 0})\end{array}$ & $\begin{array}{c}\text { Non-PCGP WNT } \\
(\mathbf{n}=\mathbf{3 9})\end{array}$ & $\begin{array}{c}\text { PCGP SHH } \\
(\mathbf{n}=\mathbf{1 1})\end{array}$ & $\begin{array}{c}\text { Non-PCGP SHH } \\
(\mathbf{n}=\mathbf{3 7})\end{array}$ \\
\hline CTNNB1 & $8(80 \%)$ & $38(97 \%)$ & $0(0 \%)$ & $0(0 \%)$ \\
\hline APC & $1(10 \%)$ & $1(3 \%)$ & $0(0 \%)$ & $0(0 \%)$ \\
\hline PTCH1 & $0(0 \%)$ & $2(5 \%)$ & $3(25 \%)$ & $15(41 \%)$ \\
\hline TP53 & $0(0 \%)$ & $4(10 \%)$ & $3(25 \%)$ & $7(19 \%)$ \\
\hline DDX3X & $4(40 \%)$ & $14(36 \%)$ & $1(8 \%)$ & $8(22 \%)$ \\
\hline BRCA2 & $0(0 \%)$ & $0(0 \%)$ & $1(8 \%)$ & $1(3 \%)$ \\
\hline PTEN & $0(0 \%)$ & $0(0 \%)$ & $1(8 \%)$ & $3(8 \%)$ \\
\hline SUFU & $0(0 \%)$ & $0(0 \%)$ & $1(8 \%)$ & $1(3 \%)$
\end{tabular}

NOTE. Comparison of most common mutations as first identified in PCGP with expanded cohort of patients with WNT and SHH.

Abbreviations: PCGP, Pediatric Cancer Genome Project; SHH, Sonic Hedgehog; WNT, Wingless.

\section{SHH Subgroup}

The majority of SHH tumors (35 of $48,73 \%$ ) were located within a cerebellar hemisphere, whereas the remaining (13 of $48,27 \%$ ) were midline. Eight (17\%) were high risk, all with $M_{+}$disease. Fourteen (29\%) had a disease recurrence. The pattern of recurrence was local in six (43\%), distant in seven (50\%), and combined local and distant in one (7\%). One second malignancy, a fatal high-grade glioma in a patient with Li-Fraumeni syndrome, was observed (Data Supplement).

The most commonly altered genes were PTCH1 (38\%), TP53 (21\%), DDX3X (19\%), and MYCN (17\%) (Fig 3C). Common chromosomal alterations included 9q loss (53\%), $17 p$ loss (26\%), and 10q loss (21\%) (Fig 3C). Mutational frequencies were similar between the initial PCGP SHH cohort $(n=11)$ and the expanded (non-PCGP) SHH cohort $(\mathrm{n}=37$ ) (Table 2, all $P>.10) .{ }^{16}$ Mutations in ELPI (7 in $48,15 \%$ ) were exclusive to $\mathrm{SHH}$ tumors, five of which were confirmed to be germline (Fig 3C). ${ }^{20}$

In univariable analyses, $\mathrm{M}_{+}$disease, LC/A histology, TP53 mutation, MYCN amplification, GLI2 amplification, and chromosome $17 p$ loss were all associated with poor PFS, whereas chromosome 14q loss was equivocal $(P=.06)$ (Data Supplement).

\section{Group 3 Subgroup}

Thirty-three group 3 patients (51\%) were average risk and 32 (49\%) high risk. All except one high-risk patient were metastatic. Twenty-six (40\%) experienced disease recurrence: 21 (81\%) distant (16 leptomeningeal, two isolated to the CSF, and three extra-CNS [bone $=2$; liver $=1$ ]), four (15\%) local, and one (4\%) combined local and distant. No second malignancies were observed. 
Frequently mutated genes included SMARCA4 (15\%) and KBTBD4 (5\%). Fifteen percent harbored MYC amplification and 6\% MYCN amplification. GFII and GFIIB overexpression was observed in $15 \%$ of tumors evaluated by gene expression array. Common chromosomal alterations included isochromosome $17 \mathrm{q}(27 \%)$, chromosome $16 q$ loss (43\%), and chromosome 10q loss (33\%) (Fig 3C).

In univariable analyses, patients with $\mathrm{M}_{+}$disease and/or MYC amplification experienced a worse outcome compared with patients lacking these features (Data Supplement). No association with outcome was detected based on histology, MYCN amplification, chromosome 11 loss, chromosome 17 gain, or isochromosome 17q (Data Supplement).

\section{Group 4 Subgroup}

Eighty-seven (63\%) group 4 patients were average-risk and 52 (37\%) high-risk. All high-risk patients were metastatic. Disease recurred in $24(17 \%)$ patients: 20 (83\%) distant (17 in the CNS and 3 extra-CNS [bone marrow, soft tissue lower extremity, and femur]), three (13\%) local, and one (4\%) combined local and distant. Three patients developed second malignancies: an atypical meningioma, a fatal esthesioneuroblastoma, and a fatal high-grade glioma (Data Supplement).

The most common genetic abnormalities were amplification of OTX2 (8\%) and MYCN (7\%) and mutations in KDM6A (8\%) and TBR1 (7\%) (Fig 3C). Frequent chromosomal alterations included isochromosome 17q (55\%), chromosome 8 loss (39\%), chromosome 7 gain (31\%), chromosome 11 loss (14\%), and chromosome 18 gain (20\%).

In univariable analyses, $M_{+}$disease was associated with a worse outcome (Data Supplement). No association with outcome was detected for histology, MYCN amplification, chromosome 11 loss, chromosome 17 gain, or isochromosome 17q (Data Supplement).

\section{Outcomes by Molecular Subtype}

Methylation analysis further classified the $\mathrm{SHH}$ subgroup into known subtypes ${ }^{21,22}$ : $\mathrm{SHH} \alpha(n=32), \mathrm{SHH} \beta(n=4)$, SHHס $(n=12)$, and $\mathrm{SHH} \gamma(n=0)$ (Data Supplement). SHH $\alpha$ subtype tumors (67\%) harbored recurrent genetic alterations in PTCH1, ELP1, TP53, MYCN, and GLI2 and were characterized by frequent isochromosome 9 , chromosome $10 q$ loss, and chromosome 17p loss (Data Supplement). There was no significant difference in outcome among SHH subtypes ( $P=.5579$; Fig 4G).

Group 3 and group 4 subgroup patients were further classified into eight subtypes based on methylation analysis $^{14}$ : subtype I $(n=13)$, subtype II $(n=28)$, subtype III $(n=18)$, subtype IV $(n=8)$, subtype V $(n=19)$, subtype VI ( $n=17)$, subtype VII $(n=50)$, and subtype VIII $(n=51)$ (Data Supplement). Subtypes II, III, and IV were nearly exclusively made up of group 3 tumors, subtypes VI, VII, and VIII were predominantly group 4 tumors, and subtypes I and $V$ contained a mixture of both subgroups (Data
Supplement). Recurrent genetic events by subtype included OTX2 amplification (subtype I), MYC amplification (subtype II), SMARCA4 mutation (subtypes II and III), MYCN amplification (subtypes $\mathrm{V}$ and $\mathrm{VI}$ ), and KDM6A, ZMYM3, KMT2C, and TBR1 mutations (subtype VIII) (Data Supplement). Age, histology, sex, and metastatic status differed among group 3 and group 4 subtypes (Data Supplement). There were also differences in PFS among group 3 and group 4 patients by subtype $(P<.0001$; Fig $4 \mathrm{H}$ ). Subtype III patients had the worst outcomes, with a 5 -year PFS of $33.3 \%(95 \% \mathrm{Cl}, 17.3$ to 64.1$)$. Subtype VII patients had the best outcomes, with a 5-year PFS of $92.0 \%$ (95\% Cl, 84.7 to 99.8).

\section{Molecularly Informed Risk Classification}

We analyzed potential risk factors and modeled outcomes based on pertinent risk features. No modeling was performed on the WNT subgroup patients because of their excellent outcome. Since metastatic status was consistently associated with an inferior outcome for all non-WNT subgroups, metastatic disease was retained as a high-risk feature and additional risk factors were analyzed.

For the SHH subgroup, TP53 mutation, LC/A histology, MYCN amplification, GLI2 amplification, and chromosome $17 p$ loss were all significantly associated with an increased risk in univariable models (Fig 5A, Data Supplement). Hence, when these risk factors were combined together with metastatic disease, the difference in PFS of SHH patients with $(n=25)$ versus without $(n=22)$ any of these features was significant (hazard ratio $[\mathrm{HR}]=23.2[95 \% \mathrm{Cl}$, 3.0 to 176.0$] P<.0001$ ) and identified a new high-risk and a new low-risk group, respectively (Fig 5B).

Group 3 and group 4 tumors were combined for this analysis, given the challenges associated with their confident discrimination and overlapping biology (Fig 3B)..$^{14,23}$ Subtype III and MYC amplification were adverse risk factors, whereas subtype VII was associated with a favorable outcome (Fig 5C). MYCN amplification, chromosome 11 loss, chromosome 17 gain, and isochromosome 17q were not associated with adverse risk. Thus, three risk groups were identified: a low-risk group (patients with $\mathrm{M}_{0}$ and subtype $\mathrm{VII}$ ), an intermediate-risk group (patients with $\mathrm{M}_{\mathrm{O}}$ and subtype not within III or VII), and a highrisk group (patients with $\mathrm{M}_{+}$disease or subtype III or MYC amplified) ( $\mathrm{HR}=0.3$ [95\% Cl, 0.09 to 1.1] low- vintermediaterisk group; $\mathrm{HR}=2.6[95 \% \mathrm{Cl}, 1.4$ to 4.7$]$ high- $v$ intermediaterisk group; $P<.0001$ ) (Fig 5D).

\section{DISCUSSION}

In this study, outcomes for children treated with SJMBO3 therapy were as favorable as those from previous prospective studies. ${ }^{1-5}$ Importantly, these results were achieved using the smallest radiation clinical target volume margin $(1 \mathrm{~cm})$ and the lowest cumulative doses of vincristine $\left(8 \mathrm{mg} / \mathrm{m}^{2}\right)$ and cisplatin $\left(300 \mathrm{mg} / \mathrm{m}^{2}\right)$ of any contemporary trial. Conversely, relative to contemporary trials, SJMBO3 


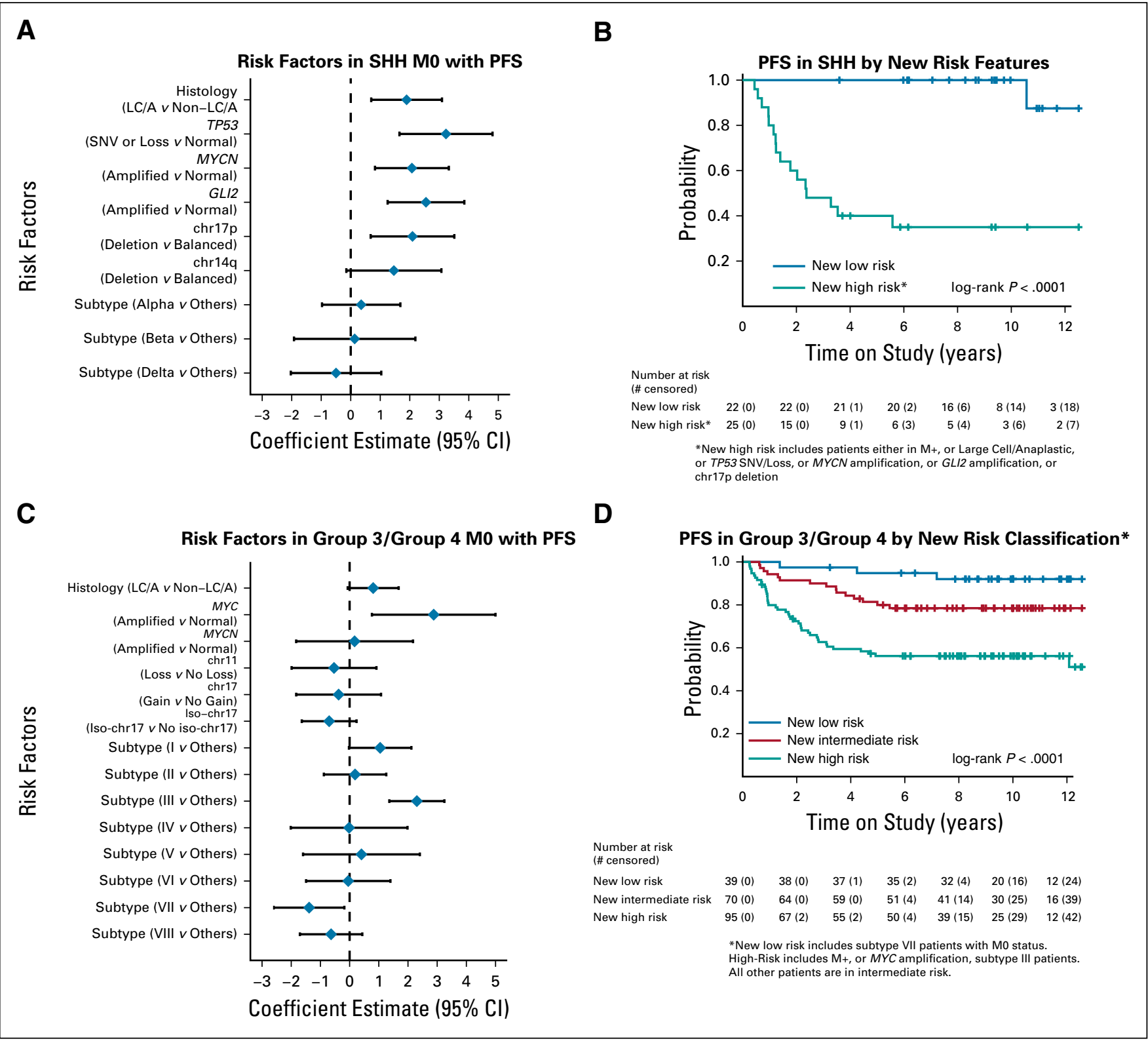

FIG 5. Risk factor modeling and survival analysis by new risk classification: (A) Forest plot displaying Log-HR and $95 \% \mathrm{Cl}$ estimates for clinical and demographic risk factors for PFS for $\mathrm{M}_{0} \mathrm{SHH}$ medulloblastoma estimated by univariable Cox models. (B) Kaplan-Meier PFS estimates by proposed new risk classification for SHH medulloblastoma where new high risk includes patients with any of the following: $\mathrm{M}_{+}$, large cell/anaplastic, TP53 SNV/Loss, MYCN amplification, GL12 amplification, or chr17p deletion; the new low risk includes patients who lack all these risk factors. (C) Forest plot displaying Log-HR and 95\% Cl estimates for clinical and demographic risk factors for PFS of the combined cohort of group 3 and group $4 \mathrm{M}_{0}$ medulloblastoma estimated by univariable Cox models. (D) Kaplan-Meier PFS estimates by proposed new risk classification for the combined cohort of group 3 and group 4 medulloblastoma where new low risk is limited to $\mathrm{M}_{0}$ subtype VII patients and new high risk includes patients who are $\mathrm{M}_{+}$, have MYC amplification, or have subtype III tumors. All other patients are in new intermediate risk. HR, hazard ratio; PFS, progression-free survival; SHH, Sonic Hedgehog; SNV, single nucleotide variant.

therapy used a high dose of CSI (36 $\left.\left[\mathrm{M}_{0-1}\right]-39.6\left[\mathrm{M}_{2-3}\right] \mathrm{Gy}\right)$ for high-risk patients and a high cumulative dose of cyclophosphamide $\left(16 \mathrm{~g} / \mathrm{m}^{2}\right)$. Still, none of these variations produced a significant difference in outcome. Taken together, this suggests that differences in outcome between contemporary medulloblastoma therapies are marginal. As such, we reason that continued modifications to clinically defined risk-stratified therapy, without regard for molecular features, will not result in any measurable benefit. Hence, this study looked to define and inform on outcome by both clinical and molecular features.

Informatively, ERBB2 protein expression did not impact the outcome of patients with medulloblastoma overall or in the context of clinical risk, in spite of preliminary data that suggested ERBB2 overexpression was an adverse risk factor. ${ }^{8,11}$ 
Traditional risk features such as presence of metastatic disease, LC/A histology, and amplification of MYC and MYCN were confirmed to negatively impact survival. Also, our extent of resection analysis supported maximal surgical resection, but the lack of difference in outcome between NTR and GTR reinforced recent findings that resection of small residual tumors that carry a high neurologic risk is of little to no benefit. ${ }^{24}$

However, most importantly, we showed methylation-based subgrouping and subtyping yielded a more precise risk profile and revealed that many of these traditional features are inextricably linked with the molecular composition of the tumor.

SJMB03 treatment achieved excellent tumor control in patients with WNT. This result substantiates the findings of many other studies, ${ }^{1,25,26}$ and the superb outcome of $>50$ patients treated uniformly on a clinical trial across multiple institutions reinforces a deintensification strategy for this subgroup. Radiation reduction has already begun for patients with WNT in three currently accruing clinical trials (SJMB12, ClinicalTrials.gov identifier: NCT01878617; PNET5, ClinicalTrials.gov identifier: NCT02066220; and ACNS1422, ClinicalTrials.gov identifier: NCT02724579).

Patients with $\mathrm{SHH}$ had the most dichotomous outcomes of all subgroups. Although survival was significantly different by clinical risk, there were other features that, when factored in post hoc, subdivided the SHH subgroup into very low-risk and very high-risk categories. Previous publications have postulated that molecular events such as TP53 mutation, LC/A histology, chromosome $17 p$ loss, and GLI2 and MYCN amplifications are interconnected, ${ }^{27,28}$ making it impossible in this relatively small cohort to determine whether one high-risk feature outperformed another. Nevertheless, patients with any of these features, including metastatic disease, had an extremely poor prognosis, whereas patients without had a remarkably favorable outcome.

For group 3 and group 4 medulloblastomas, we took multiple approaches to analyzing outcomes and risk factors.

\section{AFFILIATIONS}

${ }^{1}$ Division of Neuro Oncology, Department of Oncology, St Jude Children's Research Hospital, Memphis, TN

2Division of Brain Tumor Research, Department of Developmental Neurobiology, St Jude Children's Research Hospital, Memphis, TN ${ }^{3}$ Department of Biostatistics, St Jude Children's Research Hospital, Memphis, TN

${ }^{4}$ Department of Radiation Oncology, St Jude Children's Research Hospital, Memphis, TN

${ }^{5}$ Texas Children's Cancer Center, Baylor College of Medicine, Houston, TX

${ }^{6}$ Department of Radiation Oncology, Mayo Clinic, Rochester, MN ${ }^{7}$ Department of Pediatrics, Hospital for Sick Children, University of Toronto, Toronto, ON

${ }^{8}$ Oncology Department, Queensland Children's Hospital and University of Queensland, Brisbane, Queensland, Australia

'Department of Pathology, Royal Brisbane and Women's Hospital, University of Queensland, Brisbane, Queensland, Australia
Consistent with previous reports, patients with group 3 tumors had a significantly worse outcome than group 4 in both the average-risk and high-risk categories. ${ }^{26,29}$ MYC amplification was prognostic in group 3, but these events typically occurred among patients with metastatic disease, rendering $M Y C$ genomic status no more prognostic than metastatic status. Upon post hoc introduction of additional previously implicated risk features, we were unable to identify additional low- or high-risk features that could better stratify these patients. LC/A histology, MYCN amplification, chromosome 11 loss, chromosome 17 gain, and isochromosome $17 q$ were not prognostic in either subgroup. Thus, these findings led us to evaluate a joint clinical and subtype-driven approach that resulted in the identification of three new risk groups among combined group 3 and group 4 patients: low (patients with $\mathrm{M}_{0}$ and subtype VII), intermediate (patients with $\mathrm{M}_{\mathrm{O}}$ and subtype I, II, IV, V, $\mathrm{VI}$, and $\mathrm{VIII}$ ), and high (patients with $\mathrm{M}_{+}$disease or subtype III or MYC amplified). These groups will enable subsequent clinical trials to test judicious dose reductions on the lowest risk group (ie to cyclophosphamide and CSI), optimize therapy for the intermediate-risk patients by harmonizing the lowest doses of agents from contemporary trials to mitigate long-term sequela, and advance needed experimental therapeutics on the highest risk patients.

Nonetheless, caution should be used before adopting these stratifications. Although SJMBO3 enrolled a relatively large cohort, the survival modeling remains limited by small numbers and tumor heterogeneity, especially now that medulloblastoma consists of four molecular subgroups and 12 subtypes of disease. ${ }^{14}$ Thus, these models need to be validated on independent trial cohorts.

In conclusion, this study demonstrates the limitations of clinically defined risk stratification and highlights the power and potential of employing a combined molecular and clinical risk stratification to improve medulloblastoma therapy for all.

${ }^{10}$ Preston A. Wells Center for Brain Tumor Therapy and the Departments of Neurosurgery and Pediatrics, UF Health Shands Hospital, Gainesville, FL

${ }^{11}$ Division of Pediatric Oncology, Department of Pediatrics, Duke University, Durham, NC

${ }^{12}$ Children's Cancer Center, Royal Children's Hospital, Murdoch Children's Research Institute, Department of Pediatrics, University of Melbourne, Melbourne, Victoria, Australia

${ }^{13}$ Peter MacCallum Cancer Center, Sir Peter MacCallum Department of Oncology, University of Melbourne, Melbourne, Victoria, Australia

${ }^{14}$ The Children's Hospital at Westmead, Sydney and Division of Child and Adolescent Health, University of Sydney, Sydney, Australia

${ }^{15}$ Kids Cancer Centre, Sydney Children's Hospital, High Street, Randwick and School of Women's and Children's Health, UNSW, Sydney, Australia

${ }^{16}$ Division of Oncology, Department of Pediatrics, Children's Hospital of Philadelphia, University of Pennsylvania, Perelman School of Medicine, Philadelphia, PA 
${ }^{17}$ Department of Pharmaceutical Sciences, St Jude Children's Research Hospital, Memphis, TN

${ }^{18}$ Division of Hematology-Oncology, Department of Pediatrics, UPMC Children's Hospital of Pittsburgh, University of Pittsburgh, Pittsburgh, PA

${ }^{19}$ Omics IT and Data Management Core Facility (W610), German Cancer Research Center (DKFZ), Heidelberg, Germany

${ }^{20}$ Department of Pathology, St Jude Children's Research Hospital,

Memphis, TN

${ }^{21}$ Hartwell Center, St Jude Children's Research Hospital, Memphis, TN

${ }^{22}$ Department of Neurosurgery, College of Medicine, University of Tennessee, Memphis, TN

${ }^{23}$ Department of Bone Marrow Transplantation and Cellular Therapy, St Jude Children's Hospital, Memphis TN

${ }^{24}$ Hopp Children's Cancer Center (KiTZ), Division of Pediatric Neurooncology, German Consortium for Translational Cancer Research (DKTK), German Cancer Research Center (DKFZ), Department of Pediatric Hematology and Oncology, Heidelberg, Germany

${ }^{25}$ Department of Oncology, Cambridge Cancer Center, CRUK Cambridge Institute, Li Ka Shing Center, Cambridge, United Kingdom

\section{CORRESPONDING AUTHOR}

Giles W. Robinson, MD, St. Jude Children's Research Hospital, Department of Oncology, Division of Neuro Oncology, 262 Danny Thomas Place, MS 260, Memphis, TN 38105; Twitter: @StJudeResearch; e-mail: giles.robinson@stjude.org.

\section{EQUAL CONTRIBUTION}

A.G., G.W.R., D.W.E., and P.A.N. contributed equally to this work.

\section{SUPPORT}

Supported by the American Lebanese Syrian Associated Charities, St Jude Children's Research Hospital, NCl Cancer Center Grant (P30CA021765), Sontag Foundation (P.A.N.), Alexander and Margaret Stewart Trust (P.A.N.), American Association for Cancer Research (P.A.N.), The Brain Tumor Charity (P.A.N., G.W.R.), St. Baldrick's Foundation (P.A.N.), and Musicians Against Childhood Cancer (A.G.).

\section{CLINICAL TRIAL INFORMATION} NCT00085202

\section{AUTHORS' DISCLOSURES OF POTENTIAL CONFLICTS OF INTEREST}

Disclosures provided by the authors are available with this article at DOI https://doi.org/10.1200/JC0.20.01372.

\section{AUTHOR CONTRIBUTIONS}

Conception and design: Amar Gajjar, Giles W. Robinson, Thomas E. Merchant, Stewart J. Kellie, Richard Cohn, Matthew J. Krasin, Clinton F. Stewart, Richard J. Gilbertson, Arzu Onar-Thomas, Paul A. Northcott Financial support: Amar Gajjar, Giles W. Robinson, Paul A. Northcott Collection and assembly of data: Amar Gajjar, Giles W. Robinson, Kyle S. Smith, Thomas E. Merchant, Murali Chintagumpala, Anita Mahajan, Eric Bouffet, Ute Bartels, Tim Hassall, Thomas Robertson, Wayne Nicholls, Sridharan Gururangan, Kristin Schroeder, Greg Wheeler, Jordan R. Hansford, Stewart J. Kellie, Geoffrey McCowage, Richard Cohn, Michael J. Fisher, Matthew J. Krasin, Alberto Broniscer, Ruth G. Tatevossian, Brent A. Orr, Geoff Neale, Frederick Boop, Stefan M. Pfister, Richard J. Gilbertson, David W. Ellison

Data analysis and interpretation: Amar Gajjar, Giles W. Robinson, Kyle S. Smith, Tong Lin, Thomas E. Merchant, Anita Mahajan, Jack Su, Eric Bouffet, Tal Schechter, Sridharan Gururangan, Michael Sullivan, Jordan R. Hansford, Ivo Buchhalter, Brent A. Orr, Paul Klimo, Frederick Boop, Ashok Srinivasan, Stefan M. Pfister, Richard J. Gilbertson, Arzu OnarThomas, David W. Ellison, Paul A. Northcott

Manuscript writing: All authors

Final approval of manuscript: All authors

Accountable for all aspects of the work: All authors

\section{ACKNOWLEDGMENT}

The authors thank Emily Walker and Granger Rideout from the Hartwell Center for assistance with methylation and NGS of our tumor samples; Sujuan Jia from the Diagnostic Biomarkers Shared Resource for nucleic acids extraction; Matthew Lear from the Biorepository for assistance in archiving and providing the study material; Amanda Johnson, Keri Meyer, Molly Clarke, Leigh Ann Christy, Lenzie Denzin, Kate Lyons, Annemarie McClellan, Tabatha Doyle, Aksana Vasilyeva, and Dana Wallace for providing excellent research support and data management for the study; and, especially, the children and families who were treated on the study.

\section{REFERENCES}

1. Gajjar A, Chintagumpala M, Ashley D, et al: Risk-adapted craniospinal radiotherapy followed by high-dose chemotherapy and stem-cell rescue in children with newly diagnosed medulloblastoma (St Jude Medulloblastoma-96): Long-term results from a prospective, multicentre trial. Lancet Oncol 7:813-820, 2006

2. Jakacki RI, Burger PC, Zhou T, et al: Outcome of children with metastatic medulloblastoma treated with carboplatin during craniospinal radiotherapy: A Children's Oncology Group Phase I/II study. J Clin Oncol 30:2648-2653, 2012

3. von Bueren AO, Kortmann RD, von Hoff K, et al: Treatment of children and adolescents with metastatic medulloblastoma and prognostic relevance of clinical and biologic parameters. J Clin Oncol 34:4151-4160, 2016

4. Lannering B, Rutkowski S, Doz F, et al: Hyperfractionated versus conventional radiotherapy followed by chemotherapy in standard-risk medulloblastoma: Results from the randomized multicenter HIT-SIOP PNET 4 trial. J Clin Oncol 30:3187-3193, 2012

5. Packer RJ, Gajjar A, Vezina G, et al: Phase III study of craniospinal radiation therapy followed by adjuvant chemotherapy for newly diagnosed average-risk medulloblastoma. J Clin Oncol 24:4202-4208, 2006

6. Eberhart CG, Kepner JL, Goldthwaite PT, et al: Histopathologic grading of medulloblastomas: A Pediatric Oncology Group study. Cancer $94: 552-560$, 2002

7. McManamy CS, Pears J, Weston CL, et al: Nodule formation and desmoplasia in medulloblastomas-defining the nodular/desmoplastic variant and its biological behavior. Brain Pathol 17:151-164, 2007

8. Gajjar A, Hernan R, Kocak M, et al: Clinical, histopathologic, and molecular markers of prognosis: Toward a new disease risk stratification system for medulloblastoma. J Clin Oncol 22:984-993, 2004

9. Ellison DW, Kocak M, Dalton J, et al: Definition of disease-risk stratification groups in childhood medulloblastoma using combined clinical, pathologic, and molecular variables. J Clin Oncol 29:1400-1407, 2011

10. Eberhart CG, Kratz J, Wang Y, et al: Histopathological and molecular prognostic markers in medulloblastoma: c-myc, N-myc, TrkC, and anaplasia. J Neuropathol Exp Neurol 63:441-9, 2004

11. Gilbertson RJ, Clifford SC, MacMeekin W, et al: Expression of the ErbB-neuregulin signaling network during human cerebellar development: Implications for the biology of medulloblastoma. Cancer Res 58:3932-3941, 1998

12. Taylor MD, Northcott PA, Korshunov A, et al: Molecular subgroups of medulloblastoma: The current consensus. Acta Neuropathol 123:465-472, 2012 
13. Northcott PA, Shih DJ, Peacock J, et al: Subgroup-specific structural variation across 1,000 medulloblastoma genomes. Nature 488:49-56, 2012

14. Northcott PA, Buchhalter I, Morrissy AS, et al: The whole-genome landscape of medulloblastoma subtypes. Nature 547:311-317, 2017

15. Sharma T, Schwalbe EC, Williamson D, et al: Second-generation molecular subgrouping of medulloblastoma: An international meta-analysis of group 3 and group 4 subtypes. Acta Neuropathol 138:309-326, 2019

16. Robinson G, Parker M, Kranenburg TA, et al: Novel mutations target distinct subgroups of medulloblastoma. Nature 488:43-48, 2012

17. Capper D, Jones DTW, Sill M, et al: DNA methylation-based classification of central nervous system tumours. Nature 555:469-474, 2018

18. Hovestadt V, Remke M, Kool M, et al: Robust molecular subgrouping and copy-number profiling of medulloblastoma from small amounts of archival tumour material using high-density DNA methylation arrays. Acta Neuropathol 125:913-916, 2013

19. The St Jude Cloud. https://pecan.stjude.cloud/proteinpaint/study/MB-SJMB03

20. Waszak SM, Robinson GW, Gudenas BL, et al: Germline elongator mutations in sonic hedgehog medulloblastoma. Nature 580:396-401, 2020

21. Cavalli FMG, Remke M, Rampasek L, et al: Intertumoral heterogeneity within medulloblastoma subgroups. Cancer Cell 31:737-754 e6, 2017

22. Hovestadt V, Ayrault O, Swartling FJ, et al: Medulloblastomics revisited: Biological and clinical insights from thousands of patients. Nat Rev Cancer 20:42-56, 2020

23. Hovestadt V, Smith KS, Bihannic L, et al: Resolving medulloblastoma cellular architecture by single-cell genomics. Nature 572:74-79, 2019

24. Thompson EM, Hielscher T, Bouffet E, et al: Prognostic value of medulloblastoma extent of resection after accounting for molecular subgroup: A retrospective integrated clinical and molecular analysis. Lancet Oncol 17:484-495, 2016

25. Ellison DW, Onilude OE, Lindsey JC, et al: beta-Catenin status predicts a favorable outcome in childhood medulloblastoma: The United Kingdom Children's Cancer Study Group Brain Tumour Committee. J Clin Oncol 23:7951-7957, 2005

26. Northcott PA, Korshunov A, Witt H, et al: Medulloblastoma comprises four distinct molecular variants. J Clin Oncol 29:1408-1414, 2011

27. Rausch T, Jones DT, Zapatka M, et al: Genome sequencing of pediatric medulloblastoma links catastrophic DNA rearrangements with TP53 mutations. Cell $148: 59-71,2012$

28. Kool M, Jones DT, Jager N, et al: Genome sequencing of SHH medulloblastoma predicts genotype-related response to smoothened inhibition. Cancer Cell 25: 393-405, 2014

29. Shih DJ, Northcott PA, Remke M, et al: Cytogenetic prognostication within medulloblastoma subgroups. J Clin Oncol 32:886-896, 2014 
AUTHORS' DISCLOSURES OF POTENTIAL CONFLICTS OF INTEREST

Outcomes by Clinical and Molecular Features in Children With Medulloblastoma Treated With Risk-Adapted Therapy: Results of an International Phase III Trial (SJMB03)

The following represents disclosure information provided by authors of this manuscript. All relationships are considered compensated unless otherwise noted. Relationships are self-held unless noted. I = Immediate Family Member, Inst = My Institution. Relationships may not relate to the subject matter of this manuscript. For more information about ASCO's conflict of interest policy, please refer to www.asco.org/rwc or ascopubs.org/jco/authors/author-center.

Open Payments is a public database containing information reported by companies about payments made to US-licensed physicians (Open Payments).

\section{Amar Gajjar}

Consulting or Advisory Role: Roche/Genentech

Research Funding: Genentech, Kazia Pharmaceutical, QED Therapeutics Inc

\section{Giles W. Robinson}

Consulting or Advisory Role: Lilly, Roche/Genentech

Research Funding: Novartis, Genentech/Roche, Genentech/Roche

Thomas E. Merchant

Travel, Accommodations, Expenses: Philips Healthcare

Eric Bouffet

Consulting or Advisory Role: Novartis

Research Funding: Roche, Bristol-Myers Squibb

Jordan R. Hansford

Consulting or Advisory Role: Bayer

Geoffrey McCowage

Consulting or Advisory Role: Biogen

Research Funding: Novartis, Merck

Travel, Accommodations, Expenses: BIOGEN

Michael J. Fisher

Honoraria: AstraZeneca

Research Funding: AstraZeneca, Array BioPharma, Exelixis

Travel, Accommodations, Expenses: AstraZeneca, SpringWorks

Alberto Broniscer

Consulting or Advisory Role: QED Therapeutics, Inc.

\section{Frederick Boop}

Employment: Semmes Murphey Clinic

\section{Stefan M. Pfister}

Research Funding: Lilly, Bayer, Roche, PharmaMar, Pfizer

Patents, Royalties, Other Intellectual Property: patent on utilizing DNA methylation profiling for tumor classification

\section{Richard J. Gilbertson}

Research Funding: AstraZeneca/Medlmmune

Patents, Royalties, Other Intellectual Property: Patent together with AstraZeneca/MedImmune and the University of Cambridge for the C11orf95RELA diagnostic antibody

Arzu Onar-Thomas

Consulting or Advisory Role: Roche

Research Funding: Novartis, Apexigen, Pfizer, Celgene, Merck, Novocure Travel, Accommodations, Expenses: Roche

David W. Ellison

Patents, Royalties, Other Intellectual Property: Sole inventor of U.S. Patent No. 9,005,907 issued April 14, 2015 "Methods and Compositions for Typing Molecular Subgroups of Medulloblastoma", 62627291/S88435 1190US.P1 February 2018 "Epigenetic Histone Regulation Mediated by CXorf67" published August 2019 as WO 2019/155387

No other potential conflicts of interest were reported. 


\section{University Library}

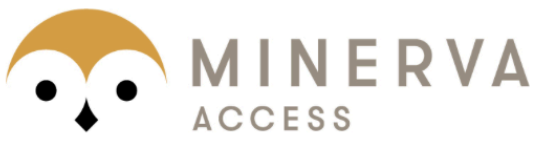

A gateway to Melbourne's research publications

Minerva Access is the Institutional Repository of The University of Melbourne

\section{Author/s:}

Gajjar, A;Robinson, GW;Smith, KS;Lin, T;Merchant, TE;Chintagumpala, M;Mahajan, A;Su, J;Bouffet, E;Bartels, U;Schechter, T;Hassall, T;Robertson, T;Nicholls, W;Gururangan, S;Schroeder, K;Sullivan, M;Wheeler, G;Hansford, JR;Kellie, SJ;McCowage, G;Cohn, R;Fisher, MJ;Krasin, MJ;Stewart, CF;Broniscer, A;Buchhalter, I;Tatevossian, RG;Orr, BA;Neale, G;Klimo, P;Boop, F;Srinivasan, A;Pfister, SM;Gilbertson, RJ;Onar-Thomas, A;Ellison, DW;Northcott, PA

Title:

Outcomes by Clinical and Molecular Features in Children With Medulloblastoma Treated With Risk-Adapted Therapy: Results of an International Phase III Trial (SJMB03)

Date:

2021-03-01

\section{Citation:}

Gajjar, A., Robinson, G. W., Smith, K. S., Lin, T., Merchant, T. E., Chintagumpala, M., Mahajan, A., Su, J., Bouffet, E., Bartels, U., Schechter, T., Hassall, T., Robertson, T., Nicholls, W., Gururangan, S., Schroeder, K., Sullivan, M., Wheeler, G., Hansford, J. R. ,... Northcott, P. A. (2021). Outcomes by Clinical and Molecular Features in Children With Medulloblastoma Treated With Risk-Adapted Therapy: Results of an International Phase III Trial (SJMB03). Journal of Clinical Oncology, 39 (7), pp.822-835. https://doi.org/10.1200/JCO.20.01372.

Persistent Link:

http://hdl.handle.net/11343/278773 